\title{
Attenuation technique for measuring sediment displacement levels
}

by R. J. Munro \& S. B. Dalziel.

Department of Applied Mathematics and Theoretical Physics, University of Cambridge, Wilberforce Road, Cambridge CB3 OWA, UK.

\begin{abstract}
A technique for obtaining accurate, high (spatial) resolution measurements of sediment redeposition levels is described. In certain regimes, the method may also be employed to provide measurements of sediment layer thickness as a function of time. The method uses a uniform light source placed beneath the layer, consisting of transparent particles, so that the intensity of light at a point on the surface of the layer can be related to the depth of particles at that point. A set of experiments, using the impact of a vortex ring with a glass ballotini particle layer as the resuspension mechanism, are described to test and illustrate the technique.
\end{abstract}

\section{Introduction}

Experimental investigations of particle resuspension and redeposition in a subaqueous environment, caused when fluid motions and structures interact with a particle layer, require techniques for obtaining accurate measurements of small-scale particle redistribution levels over a certain domain. A number of techniques are available for measuring the sediment depth levels in both field experiments and the laboratory, and in subaerial and subaqueous environments.

For field experiments the majority of researchers have employed either acoustic or laser range finding devices (e.g. Williams \& Jackson (1998) and Irish \& White (1998)). In the case of laboratory scale studies, where spatial resolution over much smaller scales is required, the current available techniques include stereo photography (Hancock \& Willgoose, 2001), infrared beams (Richards \& Robert, 1986), sonic (Richardson et al., 1961; Dingler et al., 1977) and ultrasonic (Best \& Ashworth, 1994) depth profilers, and laser 3D-scanning devices (Lague et al., 2003). These techniques, however, are subject to several limitations. For instance, sonic/ultrasonic depth profilers and stereo-photography techniques offer only limited spatial resolution (stereo-photography is also sensitive to shadow effects), while laser scanning devices are expensive and typically require (relatively) long acquisition times to scan a given sample area (e.g. the array of telemetric lasers used in Lague et al. (2003) required 60 secs to scan a sample area of dimensions $20 \mathrm{~cm} \times 30 \mathrm{~cm}$ ). (Note that, Lague et al. (2003) is specifically applied in a subaerial environment; however, with suitable modifi- 
cations it should be possible to apply this method in a subaqueous environment (Lague, 2004).)

Temporal measurements of sediment depth have been made in a subaqueous environment by measuring the electrical resistance of the particle layer using an array of electrodes in the base of the tank beneath layer (Rooij et al., 1999). With a reference electrode placed in the water above the layer, the resistance of the particles above each of the base electrodes can then be measured, and used to calculate the layer depth at that point. This method is limited in that (a) the voltage signal becomes saturated for deep particle layers, hence limiting the dynamic range of the measurements, and (b) only limited spatial and temporal resolution is available with current systems.

Munro et al. (2004) proposed a novel, high accuracy technique which involved projecting a sequence of different random patterns onto an initially undisturbed particle layer. Comparing images of the projected patterns before and after the particles had been redistributed (viewed at an angle oblique to that of the projection) allows the use of a high accuracy pattern matching algorithm to provide high resolution particle redeposition measurements.

The pattern matching technique (like the method to be described in this article) was specifically designed for the purpose of analyzing the resuspension and redeposition produced by the impact of a vortex ring with a particle layer (Munro \& Dalziel, 2003). However, the nature of the required experimental setup means that the measurements produced will be significantly disrupted as soon as particles are resuspended into the fluid above the layer. Hence, despite the fact that, in theory, the technique is capable of measuring temporal changes in the layer depth, it was found impractical to employ the method for this purpose. This meant that the pattern matching method was restricted to providing measurements of the final impact area after all resuspended particles had returned to rest.

Of course, measurements of the final state are an essential aspect of this study. However, it is also necessary to be able to quantify the temporal development of the impact site, and in particular, resolve to short time scales over which the initial layer deformation occurs.

This paper presents a non-intrusive light attenuation technique which, like the pattern matching method, is capable of providing accurate high-resolution measurements of the final particle layer state. In addition, the technique is also able to provide high temporal resolution measurements of the initial layer deformation prior to the actual resuspension event.

The method is based on the way in which light, produced from a (uniform) source placed beneath the particle layer, is able to penetrate through the (transparent) particles within the layer. That is, the light intensity at each point on the particle layer surface will be directly related to the depth of the layer at that 
point. This technique is analogous to the light attenuation technique describe in Leppinen \& Dalziel (2001) for measuring void fraction in a two-phase flow. The current technique is appropriate for layers consisting of small transparent particles (whose size is small in comparison with the characteristic transverse dimension of the layer deformation) with narrow size distribution whose packing is essentially homogeneous.

As in Munro et al. (2004), a set of experiments using the impact of a vortex ring with a glass ballotini particle layer as the resuspension mechanism, are described to illustrate the technique.

The details of the experimental setup are given in section 2 , followed by a description of the calibration procedure and attenuation model in section 3 . The results obtained are given in section 4, which includes a comparison with results obtained from the pattern matching technique, and an illustration of the types of time dependent characteristics which can be analyzed. The paper concludes with final discussions in section 5 .

\section{Apparatus}

Figure 1 shows the basic experimental setup. A large light bank, fitted with an array of twelve $180 \mathrm{~cm}$ high frequency $58 \mathrm{~W}$ florescent battens arranged in pairs with a $15 \mathrm{~cm}$ spacing, was positioned horizontally on the floor with the light face pointing upwards. An acrylic sheet was fixed across the light face to act as a diffuser.

Using a flat wooden platform, a Perspex tank with dimensions $30 \mathrm{~cm} \times$ $30 \mathrm{~cm} \times 40 \mathrm{~cm}$ was then fixed in a horizontal position above the light bank as shown (at a height of approximately $30 \mathrm{~cm}$ above the florescent battens). Large black-out curtains were used to cover the cabinet so that the only light emitted entered through the bottom of the tank.

The tank was then partially filled with tap water, and small glass ballotini, with size distribution $0.02-0.03 \mathrm{~cm}$ and density $\rho=2.4 \mathrm{~g} \mathrm{~cm}^{-3}$ were added and formed into a smooth, even layer of depth $z_{r}$; two values of $z_{r}$ were used in the experiments, $0.2 \mathrm{~cm}$ and $0.8 \mathrm{~cm}$. The tank was then topped up with water to a level $7 \mathrm{~cm}$ below the top of the tank.

The incident light from the uniform light source will experience multiple reflections, refractions and absorption as it penetrates the layer of transparent glass particles (with refractive index $n \approx 1.5$ ). Hence the geometry and packing of the particles within the layer will play a significant role in determining the degree of attenuation experienced by the light as it passes through the layer. By using spherical particles of small dimension (in comparison with the characteristic transverse length scale of the layer deformation we are attempting to measure) with a narrow size distribution, we may assume that the packing, 


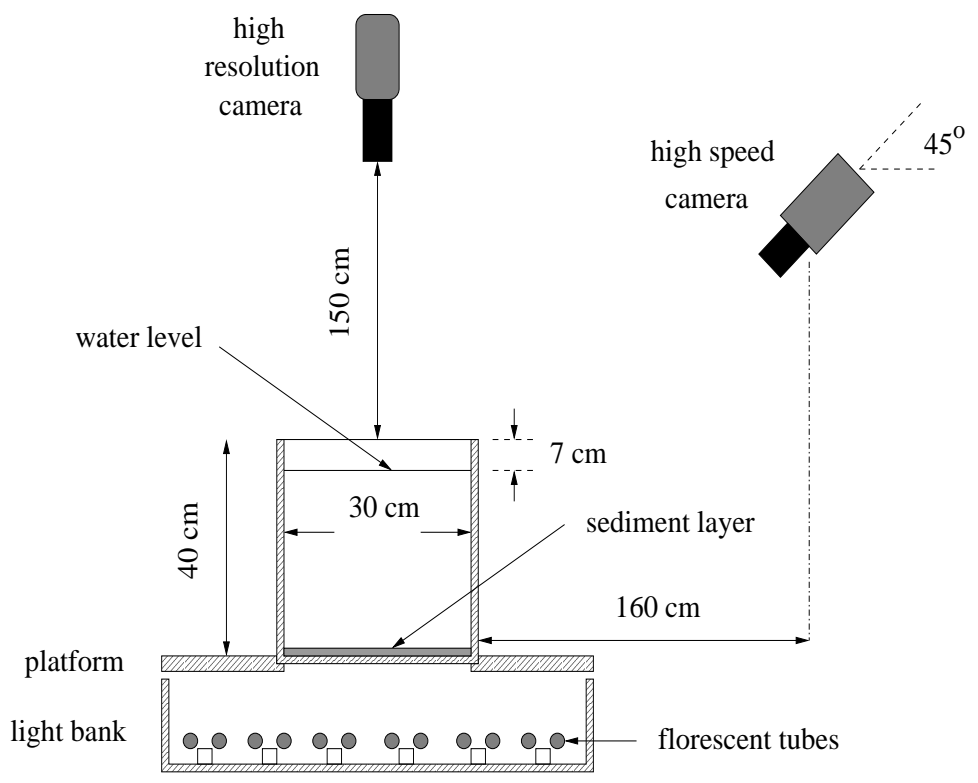

Figure 1: Setup of apparatus for the attenuation technique.

and hence surface intensity is essentially statistically homogeneous for a given uniform layer depth. This will certainly be the case in situations when the layer depth is much greater than the characteristic particle dimension, and in this case we expect the relationship between the layer depth and surface intensity to be self-similar.

However, in the zero depth limit, and certainly for isolated (individual or groups of) particles, the assumption of homogeneous packing will no longer be valid, resulting in a break down of self-similarity in the surface intensity. For $z_{r}=0.2 \mathrm{~cm}$, where the effects of the underlying boundary are important, and for the high-energy impacts with the deeper $z_{r}=0.8 \mathrm{~cm}$ layer, complete (or near complete) layer erosion through to the tank floor is likely in certain regions of the impact area. Hence, the break down of self-similarity will need to be accounted for when modelling the attenuation. This is discussed further in section 3. It is worth noting here that, using the results obtained in Munro et al. (2004) (in which $\left.z_{r}=1 \mathrm{~cm}\right)$, a dynamic range of $0-1.1 \mathrm{~cm}$ will be required.

To obtain measurements at all (pixel resolution) locations of the impact area, the view of the camera needs to be vertically down onto the particle layer. However, the rig used to generate the vortex rings was placed directly over the tank, thus obstructing the vertical view. Hence, two separate camera setups were used during the experiments to facilitate both final-state and dynamic 
measurements.

The first of these was a high-resolution digital JAI CV-M4+ camera positioned on a boom a distance of $150 \mathrm{~cm}$ above the top of the tank and focused looking vertically down onto the particle bed. Due to the obstructed view, the high resolution camera could only be used to capture images of the final impact area (i.e. when all resuspended particles have been redeposited) after the vortex ring apparatus had been moved aside. The JAI CV-M4+ has $1380 \times 1030$ pixel resolution at 10 bits per pixel, and a sampling rate of 24 frames per second. Each pixel in the captured images corresponds to an area of approximately $0.03 \mathrm{~cm} \times 0.03 \mathrm{~cm}$ on the surface of the particle layer (i.e. approximately one particle).

To enable us to capture images of the evolution of the impact area as the vortex ring approaches and interacts with the particle layer, a second camera was positioned on a tripod a distance of $160 \mathrm{~cm}$ from the side of the tank and focused looking down onto the particle bed at an angle of $45^{\circ}$. To sufficiently resolve the short time scales over which the initial deformation of the particle layer occurs, a camera with a high frame rate was required. For this purpose a digital DALSA DA6-512 camera was used, with a frame rate of 262 frames per second and a pixel resolution of $512 \times 512$ at 8 bits per pixel. Each pixel in the resulting images corresponds to an area of approximately $0.05 \mathrm{~cm} \times 0.11 \mathrm{~cm}$ on the surface of the particle layer (an area which contains approximately eight particles). Both of the cameras were used in conjunction with Cosmicar 12.5$75 \mathrm{~mm}$ f/1.8 manual zoom lenses.

Of course, variations in camera angle will result in a variation in the observed surface intensity. That is, in the case of deep layers $z / d \gg 1$, where $z$ is the layer depth and $d$ a scattering element length scale, the transmitting light is subject to many scattering events, and so we expect the functional form of this variation to be independent of $z / d$, i.e.

$$
P\left(\frac{z}{d}, \varphi\right)=Q\left(\frac{z}{d}\right) F(\varphi)
$$

Here $P$ is the layer intensity and $\varphi$ the angle of view (defined such that $\varphi=0$ corresponds to the camera looking vertically down onto the layer, and $\varphi=\pi / 2$ for the camera view looking horizontally across the layer). In particular, for the deep layer case we expect the largest proportion of light from the (diffuse) source to be reflected/refracted vertically upwards, and for this proportion to decrease as the viewing angle $\varphi$ increases, i.e. $F$ is monotonically decreasing on $[0, \pi / 2]$. Furthermore, we expect $Q$ to be exponential in the deep layer case (see section 3). However, as we pass to the zero depth limit the self-similarity in the transmitting light field will be lost, and so we would expect the variation in $P$ with $\varphi$ to also depend on $z / d$, i.e.

$$
P\left(\frac{z}{d}, \varphi\right)=Q\left(\frac{z}{d}\right) F\left(\frac{z}{d}, \varphi\right)
$$




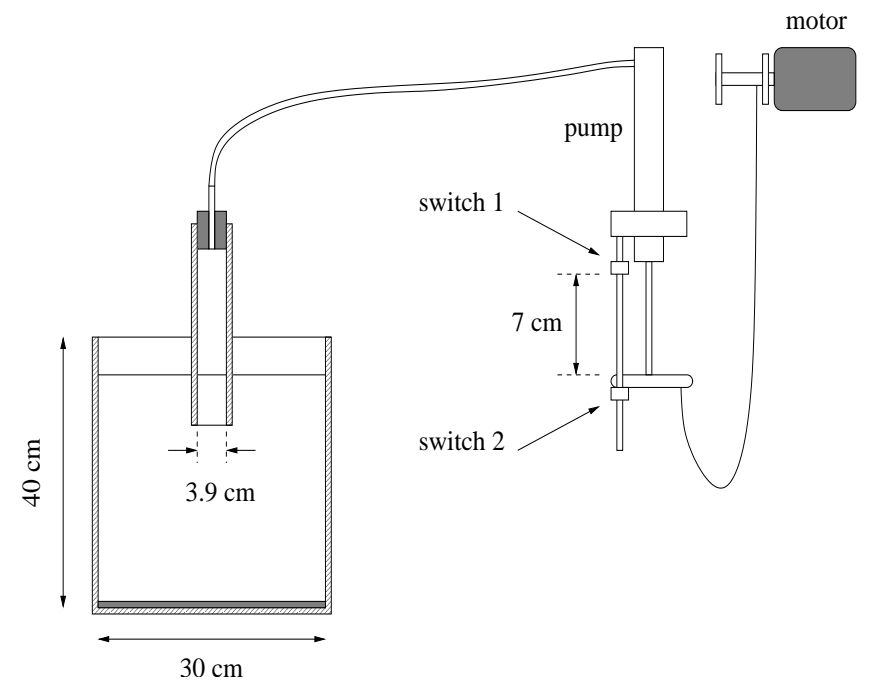

Figure 2: Schematic showing the setup for generating vortex rings.

We anticipate that such variations will be negligible for deep layers, but may become significant in the zero depth limit. Similarly, even in the case of the vertical view, variations will be produced due to the divergence in the camera view (this is discussed in more detail in section 3).

The vortex rings were generated by the same method employed in Munro et al. (2004), using a circular tube of radius $R=1.95 \mathrm{~cm}$ connected to motor driven pump with a $2.8 \mathrm{~cm}$ bore (see figure 2); a comprehensive overview of experimental formation of vortex rings can be found in Glezer (1988) and Linden \& Turner (2001). The stroke length of the pump was held constant throughout the experiments at $7 \mathrm{~cm}$, which consistently produced a fluid slug with a length of $L \approx 4 \mathrm{~cm}$, i.e. $L / 2 R \approx 1$. Note that for the limiting (inviscid) case of Hill's spherical vortex, $L / 2 R=4$ (Linden \& Turner, 2001).

The vortex ring Reynolds number, $R e=2 R U / \nu$, was determined from the stroke time $T[\mathrm{~s}]$ of the pump. (The stroke time was measured using an oscilloscope connected to two switches which could detect the start and end of the pump-handle stroke.) Once the vortex ring had propagated a distance of order $4 R$ from the orifice, the propagation speed was constant and remained so until the vortex ring was influenced by the bottom boundary a distance of $2 R$ from the tank floor; this constant propagation speed was adopted for the value of $U[\mathrm{~cm} / \mathrm{s}]$.

The initial smooth particle beds were produced by dragging over the particle layer a scraper, maintained at a constant height above the tank bottom by 
two stainless steel rods of diameter $z_{r}$ (i.e. either $0.2 \mathrm{~cm}$ or $0.8 \mathrm{~cm}$ ) located along two opposing sides of the tank. The bedforms produced had a consistent mean thickness, although invariably were not completely flat. The remaining features had a typical length scale of order of several centimetres in the plane of the bed, and depth of order comparable to the particle diameter. The small amplitude and long wavelength of the initial irregularities did not affect the dynamics of the vortex ring or the resuspension.

Once the particle layer had been prepared, the tube was fixed vertically in the centre of the tank with the outlet a depth of $7 \mathrm{~cm}$ below the water surface. The motorized pump was set to a known stroke time and the motor activated, propelling a vortex ring towards the particle layer. At the same time, the high-speed camera began to capture images, and was stopped after the impact only when all the resuspended material had been redeposited. The tube was then removed so an image of the impact area could be taken using the highresolution camera ${ }^{1}$. This was repeated for a range of Reynolds numbers between $8000 \leqslant R e \leqslant 29000$ for each of the two layer depths $z_{r}$. For the particles used here, no resuspension was produced by the impact for $R e \leqslant 5000$.

It is worth noting here that the accuracy of this method will be assessed by a direct comparison between measurements produced from the images captured using the high-resolution camera, and a corresponding set of measurements produced from the pattern matching technique described in Munro et al. (2004), obtained from an essentially identical set of experiments. In Munro et al. (2004) a manufactured synthetic crater with a known profile, made to simulate the features of the impact areas observed in the experiments, was used to quantify the accuracy of the pattern matching technique. The results of this procedure showed that in measuring displacements of the order of $\pm 0.3 \mathrm{~cm}$, the corresponding error associated with the pattern matching method was always within $0.03 \mathrm{~cm}$ (which corresponds to approximately one particle diameter).

\section{Calibration}

Let $\left(x_{p}, y_{p}\right)$ denote the image coordinates in (continuous) pixel space, and $P\left(x_{p}, y_{p}, t_{i}\right)$ the set of pixel intensities in each image frame at time step $t_{i}$. These intensities are scaled in the range $0 \leqslant P\left(x_{p}, y_{p}, t_{i}\right) \leqslant 1$. Note that, both of the cameras used in the experiments exhibit a linear response with a small background offset factor. For example, with the JAI CV-M4+ camera, black digitizes to 0.035 . Hence, prior to any calibration or processing procedures, this small offset factor is first subtracted from each $P\left(x_{p}, y_{p}, t_{i}\right)$.

\footnotetext{
${ }^{1}$ Note that, since the view of the high-resolution camera is vertically down into the tank, any disturbances to the free surface will result in a distortion of the captured images. Hence, before each image is taken care was taken to allow the free surface to become stationary.
} 
The aim of the calibration is to determine an empirical relationship to describe how the intensity field, $P\left(x_{p}, y_{p}, t_{i}\right)$, corresponding to an element of the particle layer surface depends on the depth of the layer, $z\left(x_{p}, y_{p}\right)$, above tank floor. This was achieved in the following way; note that same procedure described below was adopted for each of the two camera views.

First, with no particles in the tank, a sequence of the illuminated tank floor was captured; in each case the sequence consisted of twenty frames. To reduce inherent noise the sequence was time-averaged to produce the image $\bar{P}\left(x_{p}, y_{p} ; z_{0}\right)$, where $z_{0}$ denotes the zero reference depth. The aperture of the camera was adjusted and set so that the maximum value of $\bar{P}\left(x_{p}, y_{p} ; z_{0}\right)$ was just below 1 (i.e. the image was not saturated).

Using the scraper method outlined in section 2, pairs of stainless steel rods with appropriate diameters were used to set the level of the particle layer in the bottom of the tank to five further reference heights: $z_{1}=0.2 \mathrm{~cm}, z_{2}=0.5 \mathrm{~cm}$, $z_{3}=0.8 \mathrm{~cm}, z_{4}=1.0 \mathrm{~cm}$, and $z_{5}=1.2 \mathrm{~cm}$. Again, a sequence of each reference layer surface was captured and time-averaged to produce the set of averaged intensity fields $\bar{P}\left(x_{p}, y_{p} ; z_{k}\right)$.

To remove any effects caused by non-uniformities in the illumination or imperfections in the base of the perspex tank, the set of scaled intensity fields,

$$
I_{k}\left(x_{p}, y_{p}\right)=\frac{\bar{P}\left(x_{p}, y_{p} ; z_{k}\right)}{\bar{P}\left(x_{p}, y_{p} ; z_{0}\right)} \quad(0 \leqslant k \leqslant 5),
$$

were generated. The subsequent analysis showed that, as hoped, for each $k$ the scaled intensity fields, $I_{k}\left(x_{p}, y_{p}\right)$, were essentially homogeneous.

Note, by definition, $I_{0}\left(x_{p}, y_{p}\right) \equiv 1$. For the vertical view from the highresolution camera we expect that $I_{k}\left(x_{p}, y_{p}\right)<I_{k-1}\left(x_{p}, y_{p}\right)$ for each $1 \leqslant k \leqslant 5$. As already mentioned, we also expect the same will be true for other angles once the particle layer is a few particle diameters thick, but may not hold for very thin layers being viewed far from vertical.

For each $I_{k}\left(x_{p}, y_{p}\right)(k \geqslant 1)$ we found the spatial average intensity $\left\langle I_{k}\right\rangle$, together with the corresponding standard deviation $\sigma_{k}$. The resulting values obtained from the vertical view of the high-resolution camera are shown in figure 3 (points) plotted along with the corresponding error bars $\left\langle I_{k}\right\rangle \pm \sigma_{k}$. It was found that, for the high-resolution camera view, $0.02<\sigma_{k} /\left\langle I_{k}\right\rangle<0.05$, and $0.03<\sigma_{k} /\left\langle I_{k}\right\rangle<0.05$ for the high-speed camera view, and in both cases, as expected, $\sigma_{k}$ increased in the zero depth limit (see figure 3 ).

Hence, we shall assume that surface intensity variations due to statistical inhomogeneity in the zero depth limit are negligible, and hence that the scaled intensity $I(z)$ is independent of $\left(x_{p}, y_{p}\right)$. The measurements shown in figure 3 suggest that the attenuation of $I(z)$ can be represented by,

$$
\frac{I(z)}{I_{0}}=\exp \left\{\alpha\left(\frac{z}{d}\right)^{p}\right\}
$$




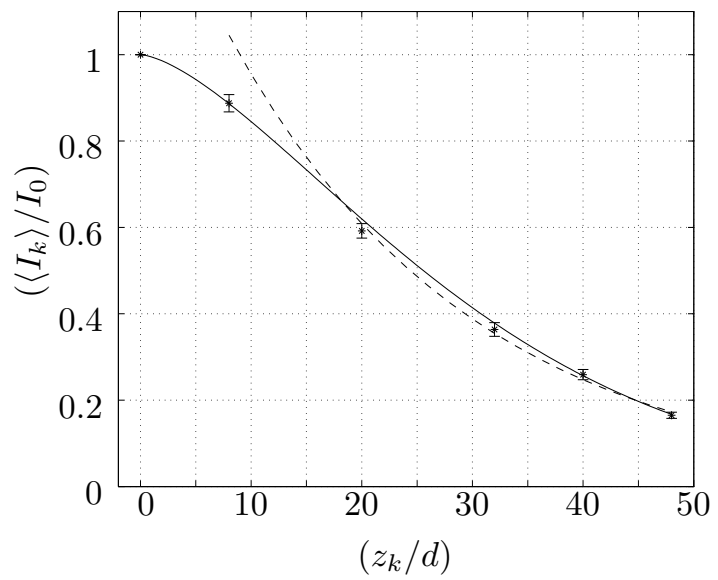

Figure 3: Plot showing the values of $\left\langle I_{k}\right\rangle / I_{0}$ (points) obtained from the high-resolution camera, against nondimensionalized depth, $z_{k} / d$, where $d=0.025 \mathrm{~cm}$ is the characteristic particle diameter, together with the error bars $\left\langle I_{k}\right\rangle \pm \sigma_{k}$. The solid line shows the curve given by (4) with $p=1.5067$ and $\alpha=-0.0052$ obtained using a least squares procedure. The broken line shows the curve given by (5) with $z_{s}=z_{2}$ and $\beta=-0.1801$.

where here the characteristic particle diameter $d=0.025 \mathrm{~cm}$, the length scale between scattering elements in the particle layer, has been used to nondimensionalize the layer depth $z$. Note that, for $z \geqslant 0$, to ensure the necessary conditions that $I(0)=I_{0}$ and $I(z) \rightarrow 0$ as $z \rightarrow \infty$, we require $p>0$ and $\alpha<0$ respectively. (The expression defined in equation (4) was chosen for empirical convenience in that it accurately described the data over the required range of layer depths, and is not presented with any particular physical justification.)

A least squares fit was performed to the values of $\left\langle I_{k}\right\rangle$ obtained from both the vertical and angled camera views, which produced $p=1.5067, \alpha=-0.0052$ and $p=1.2759, \alpha=-0.0073$ respectively. The curve obtained for the vertical view of the high resolution camera is shown in figure 3 (solid line). The difference in the parameter values obtained for the two camera views are mainly due to the difference in viewing angle.

Note that, as previously mentioned in section 2, once the depth of the layer is sufficiently large so that the packing within the layer is essentially statistically homogeneous and the light has undergone a number of scattering events, we expect the relationship between $I$ and $z$ to be self-similar. In particular, in this range we expect $I(z)$ to obey the Beer-Lambert law (Measures, 1984),

$$
\frac{I(z)}{I_{s}}=\exp \left\{\beta\left(\frac{z-z_{s}}{d}\right)\right\} \quad\left(z \geqslant z_{s}\right),
$$

where $z_{s}$ is layer depth above which these conditions are satisfied, and $I_{s}=$ 
$I\left(z_{s}\right)$. (Note that, $\beta / d$ is commonly known as the extinction coefficient.) The data suggests that self-similarity is obtained for depths $z \gtrsim 18 d$; this is illustrated by the broken curve in figure 3 which shows the least squares fit for (5) performed to the $\left\langle I_{k}\right\rangle$ data for $z \geqslant z_{2}(\beta=-0.1801)$, again for the vertical view camera.

In applications where the required dynamic range is inside the self-similar regime, the appropriate calibration function will be the inverted form of (5). However, if like the application described here, the dynamic range is required to include the zero depth limit then the appropriate calibration function is the inverted form of (4), i.e.

$$
z\left(x_{p}, y_{p}\right)=d\left\{\frac{1}{\alpha} \log \left(\frac{I}{I_{0}}\right)\right\}^{1 / p}
$$

At this point, it is worth briefly discussing the vertical resolution we expect to achieve in our measurements. From Munro et al. (2004), we expect to observe maximum vertical displacements, $\Delta z=\left(z-z_{r}\right)$, of approximately $10 d$, and with $z_{r} \leqslant 32 d$, it follows that the operational dynamic range will be approximately $0 \leqslant z \leqslant 42 d$. Within this range (see figure 3 ) the change in intensity corresponding to a change in layer depth of order $d, \Delta I$ say, is always much larger than either the (local) value of $\sigma_{k}$, or the noise level of the camera. For example, at the upper limit of this range at around $(z / d)=40$ (where we expect $\left|\Delta I-\sigma_{k}\right|$ to be smallest), we have $\Delta I \approx 0.013$ and $\sigma_{5} \approx 0.004$. Hence, within the operational range for our experiments, it is possible to resolve vertical displacements of the order of one particle diameter $d$. It should be noted, however, that this does not mean that the method is able to resolve the displacement of a single particle, but that it is able to detect vertical displacements in the bedform of the order of $d$. For much greater depths (i.e. for say $(z / d)>50$ ), $\Delta I$ may become of the order $\sigma_{k}$ (or, more likely, the noise level of the camera), and in this case the resolution will be reduced.

The final step of the calibration was to transform the image coordinates and intensity, $\left(x_{p}, y_{p}, I\left(x_{p}, y_{p}\right)\right)$, into the corresponding physical coordinates, $(x, y, z(x, y))$, where $(x, y)$ are the transverse coordinates in the plane of the undisturbed particle layer, $z_{r}$. In general, in a disturbed image, the diverging camera view means that the image coordinates $\left(x_{p}, y_{p}\right)$ in a region where $\Delta z \neq 0$ will correspond to physical (transverse) coordinates $(x+\Delta x, y+\Delta y)$. The magnitude of $(\Delta x, \Delta y)$ will depend on $(x, y, z)$, and on several different factors which include the distance of the camera from the base of the tank (and general camera geometry), the water depth, and refractive index of the media (in our case air and water).

For the vertical view images, the camera is positioned a height of $6280 \mathrm{~d}$ above the water surface, which is an additional height of $1380 d$ above the undisturbed particle layer (with the centre of the image corresponding to the centre 
of the tank base). Taking the refractive indices of air and water as $n_{0}=1$ and $n_{1}=1.3$ respectively, it can be easily shown that at a distance of order $300 d$ from the centre of the tank (i.e. approximately half way to the tank side), a vertical displacement of the order $\pm 10 d$ (i.e. the maximum displacements we expect to observe) will correspond to $(\Delta x, \Delta y)$ of approximately $\mp 0.3 d$. Such small differences are insignificant in comparison with the resolution of the camera (i.e. 1 pixel $\approx 1.2 d \times 1.2 d)$, and hence $(\Delta x, \Delta y)$ can be neglected for the vertical view. (With the specifications stated above, we estimate that $(\Delta x, \Delta y)$ will become comparable with the camera resolution when $\Delta z>40 d$.)

The displacements $(\Delta x, \Delta y)$ will be significant in the case of the angled view images, and in general will need to be accounted for. However, in section 4, the high-speed camera images will be used solely for the purpose of illustrating how the technique can be used to capture time dependent measurements, while the accuracy of the technique is assessed using the vertical view, high-resolution images. Specifically, the angled-view images will be used to measure the initial development of the inner crater radius (see section 4.2) which, by its nature, is defined in the plane of the initial undisturbed layer, $z_{r}$. As a result, it is not strictly necessary to account for the transverse displacements $(\Delta x, \Delta y)$ for the angled view either.

Neglecting the displacements $(\Delta x, \Delta y)$ simplifies the coordinate transformation procedure which, for both views, was obtained in the following way. A coordinate mesh (with $1 \mathrm{~cm}$ spacing) was sketched on the surface of a rigid, horizontal plastic platform, $30 \mathrm{~cm} \times 30 \mathrm{~cm} \times 0.2 \mathrm{~cm}$, which could be adjusted to any reference height between $0.2 \mathrm{~cm}$ and $1.6 \mathrm{~cm}$. The platform was then set to the $z_{r}=0.2 \mathrm{~cm}$ reference height and placed in the bottom of the tank, making sure the water depth was maintained at a depth $7 \mathrm{~cm}$ below the top of the tank. An image of the coordinate grid was captured by both of the cameras, and the relationship between $\left(x_{p}, y_{p}\right)$ and the corresponding physical coordinates $(x, y)$ in the $z_{r}$ plane established using the linear map,

$$
(x, y)=\left(\xi_{2} y_{p}+\xi_{1} x_{p}+\xi_{0}, \eta_{2} y_{p}+\eta_{1} x_{p}+\eta_{0}\right),
$$

where the coefficients $\left\{\xi_{i}, \eta_{i}\right\}$ are found using a selected number of mesh points. This procedure was repeated for the $z_{r}=0.8 \mathrm{~cm}$ reference height. Note that, in all the subsequent sections describing the processing procedure, the images will first be transformed into the relevant physical coordinate space, $(x, y)$, using (7).

In the general case, when the transverse displacements $(\Delta x, \Delta y)$ are significant and need to be accounted for, the coordinate transformation will be of the form

$$
(x, y)=\left(f_{1}\left(x_{p}, y_{p}, I\left(x_{p}, y_{p}\right)\right), f_{2}\left(x_{p}, y_{p}, I\left(x_{p}, y_{p}\right)\right)\right),
$$

where the functional forms of the $f_{1}$ and $f_{2}$ will depend on the general camera 
geometry. The above coordinate transformation can be obtained using a series of coordinate grid images (obtained using the same method described above) at different reference heights $z_{r}$ resolving the range of displacements $\Delta z$ observed.

\section{Results}

This section is divided into two subsections. The first describes how the images captured using the high-resolution camera are processed to provide measurements of the final impact crater produced in each of the experiments described in section 2. In the second subsection we describe how attenuation technique may be employed to measure temporal changes in layer depth using the high-speed camera images. It should be noted that this element of the processing is specific to the resuspension experiments used here to illustrate the attenuation technique.

We begin, however, with some comments on the validation process. As already mentioned, in Munro et al. (2004) a manufactured synthetic crater with a known profile was used to assess the accuracy of the pattern matching technique. A similar approach is not possible for the attenuation technique as the present approach relies on the passage of light through the particles, a feature that cannot be easily or accurately mimicked by a manufactured crater. Hence, a corresponding set of reference measurements, obtained from an independent set of experiments with $z_{r}=0.2 \mathrm{~cm}$, using the pattern matching method will be used for a comparison (these measurements are known to be accurate within at least $0.03 \mathrm{~cm}$, i.e. approximately one particle diameter, $d$ ). It should be noted that, as the measurements were obtained from independent experiments we do not expect an exact alignment between both sets of measurements. We do, however, expect there to be close agreement between the average properties of the measured craters for each value of $R e$.

\subsection{High-resolution camera images}

The procedure described below was applied separately to the images obtained for both the $z_{r}=0.2 \mathrm{~cm}$ and $z_{r}=0.8 \mathrm{~cm}$ cases, using the appropriate physical coordinate transformation and calibration curve. For each of the crater images, as with the calibration layers, twenty frames were captured, and the resulting image time-averaged to produce the set of intensity fields $\bar{P}(x, y)$. So that the calibration function in equation (6) could be applied, the corresponding set of scaled intensity fields,

$$
I(x, y)=\frac{\bar{P}(x, y)}{\bar{P}\left(x, y ; z_{0}\right)},
$$

were generated, two examples of which, for $R e=2 \times 10^{4}$, are shown in figures 

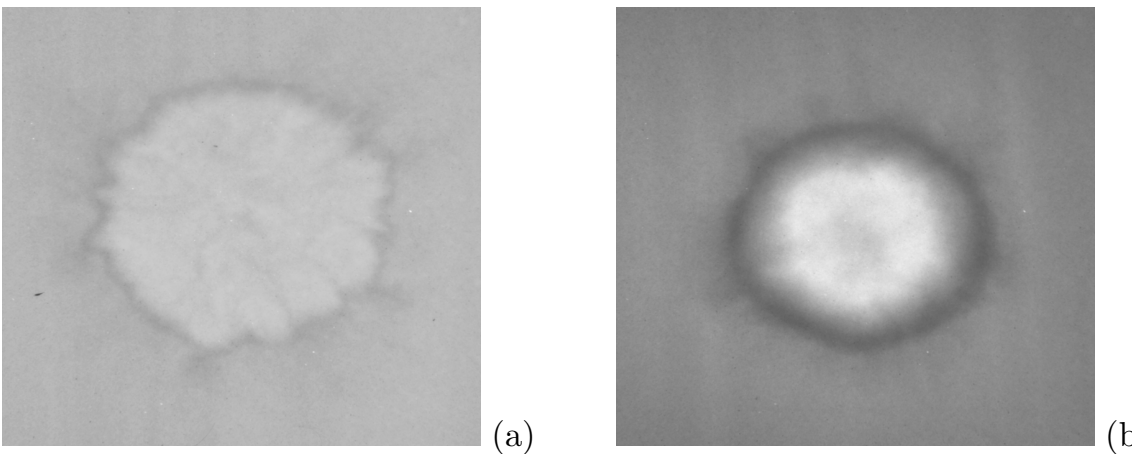

(b)

Figure 4: Examples of the scaled intensity fields $I(x, y)$ obtained from the high-resolution crater images for (a) $z_{r}=0.2 \mathrm{~cm}$, and (b) $z_{r}=0.8 \mathrm{~cm}$. In each case, the vortex ring Reynolds number was $R e=2 \times 10^{4}$.

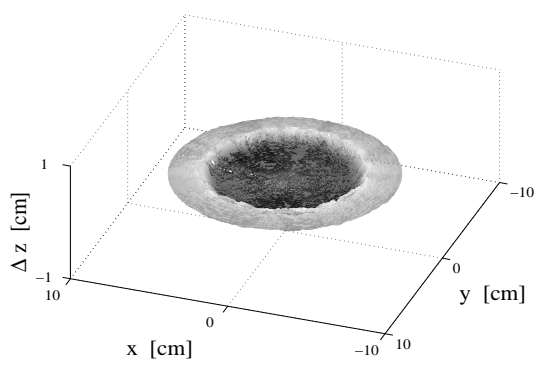

(a)

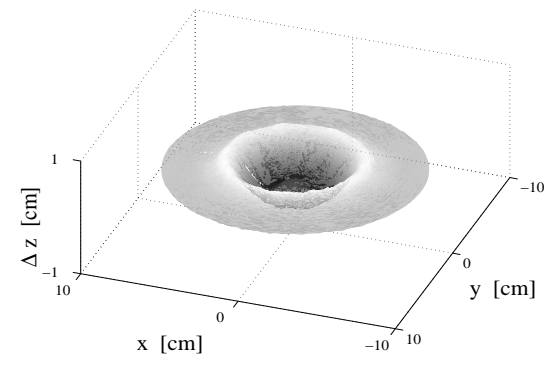

(b)

Figure 5: Plot showing the corresponding displacement fields $\Delta z(x, y)=z(x, y)-z_{r}$, for (a) $z_{r}=0.2 \mathrm{~cm}$, and (b) $z_{r}=0.8 \mathrm{~cm}$, calculated from the scaled intensity fields shown in figure 4 .

4(a) and 4(b), corresponding to $z_{r}=0.2 \mathrm{~cm}$ and $z_{r}=0.8 \mathrm{~cm}$ respectively. Obviously, in both images, the dark regions correspond to deposit and the lighter regions to erosion.

Equation (6) was applied directly to each $I(x, y)$ producing the corresponding depth field $z(x, y)$. To coincide with the reference measurements, each $z(x, y)$ obtained was translated into the corresponding displacement field, $\Delta z(x, y)$, using

$$
\Delta z(x, y)=z(x, y)-z_{r} .
$$

Typical examples of the displacement fields $\Delta z(x, y)$ obtained, corresponding to the two scaled intensity fields $I(x, y)$ in figure 4 , are shown in figure 5 .

For the $z_{r}=0.2 \mathrm{~cm}$ case, a total of five experiments were conducted, with the Reynolds number of the vortex rings used ranging between $8000 \leqslant R e \leqslant$ 
29000. For each value of $R e$, a corresponding set of reference measurements (of known accuracy) was available for comparison. As the two sets of corresponding measurements were obtained from independent experiments, albeit under essentially identical conditions, the fine scale structure of the craters produced will depend on the individual dynamics of each impact and so will be different. Of course we would expect the crater structures to have similar scales, but they will invariably have different phases in the different realizations. However, we expect the overall mean structure of the craters (in the sense of an azimuthal average) to be essentially the same.

To provide a simple mechanism for comparing the measurements obtained from the two separate techniques, each measured displacement field $\Delta z(x, y)$ was transformed into polar coordinates

$$
\begin{aligned}
& r=\sqrt{\left(x-x_{0}\right)^{2}+\left(y-y_{0}\right)^{2}}, \\
& \theta=\tan ^{-1}\left(\frac{y-y_{0}}{x-x_{0}}\right)
\end{aligned}
$$

where $\left(x_{0}, y_{0}\right)$ is the crater centre. The resultant polar displacement fields $\Delta z(r, \theta)$ were then averaged over $\theta$ to produce the corresponding set of mean (radial) profiles $\langle\Delta z\rangle$. For each value of $R e$, the mean profiles obtained using the two different techniques were compared.

The azimuthal mean profile for the displacement field shown in figure 5(a), for the $R e=2 \times 10^{4}$ impact, is shown in figure 6 (solid curve) together with the mean profile obtained from the corresponding set of reference measurements obtained using the pattern matching method (broken curve). Clearly, the correspondence is excellent with a maximum difference along the profile less than one particle diameter $d=0.025 \mathrm{~cm}$. Due to the measurements coming from independent experiments, some difference is to be expected.

Except for the two displacement fields at the lower end of the Reynolds number range, a similar excellent degree of alignment was obtained in the other profiles compared, with maximum differences of the order of a particle diameter $d$. In the two displacement fields at the lower end of the range the maximum differences between the mean profiles were typically of order $2 d-4 d$, although the overall qualitative features of the profiles were the same. At this lower end of the Reynolds number range, differences in the configuration of the particles within the layer, and indeed in the structure of the vortex rings produced between the two sets of experiments, may result in subtle differences in the precise dynamics of the impact. Hence, to some extent, the greater differences observed at this end of the range are also to be expected.

By using the synthetic profile, Munro et al. (2004) were able to estimate the accuracy of the measurements produced by the pattern matching method (i.e. in measuring maximum displacements of the order of $0.3 \mathrm{~cm}$, the degree 


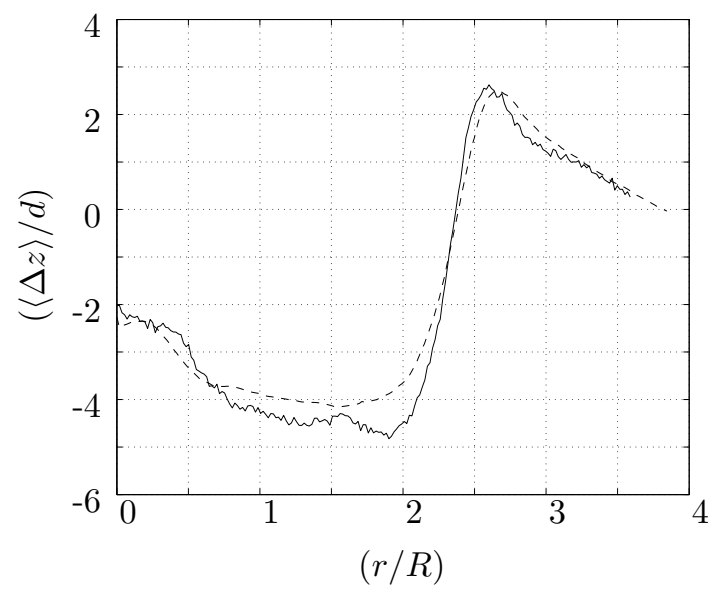

Figure 6: The mean profiles $\langle\Delta z\rangle$, nondimensionalized by the characteristic particle diameter $d=0.025 \mathrm{~cm}$, obtained from the attenuation technique experiments (solid curve) and the pattern-matching technique experiments (broken curve), for $R e=2 \times 10^{4}$. The radial coordinate $r$ has been nondimensionalized by the outlet radius $R=1.95 \mathrm{~cm}$. The solid curve is the mean profile generated from the displacement field shown in figure 5(a).

of error was always less than $0.03 \mathrm{~cm}$ ). As noted previously, a similar estimate is not possible for the attenuation method. However, the excellent degree of alignment between the two independent sets of mean profiles suggests that the attenuation method is able to provide a similar degree of accuracy as the pattern matching technique.

As an indication of the amplitude of the noise in each of the displacement fields obtained using the attenuation method, the standard deviation, $\sigma_{z}$, of $\Delta z(r, \theta)$, about the mean profile $\langle\Delta z\rangle$, was calculated from regions far from the impact zone corresponding to zero displacement. Over the range of experiments performed, with maximum measured displacements between $0.05-0.3 \mathrm{~cm}$, it was found that $0.008 \mathrm{~cm} \leqslant \sigma_{z} \leqslant 0.011 \mathrm{~cm}$. Using the maximum displacements as a measure of the signal strength, $S$, the range of corresponding signal-to-noise ratios, $S / \sigma_{z}$, calculated form each of the experiments, was $5 \leqslant\left(S / \sigma_{z}\right) \leqslant 38$. In Munro et al. (2004), application of the pattern matching technique to the known profile returned typical values for $\sigma_{z}$ in the range $0.001 \mathrm{~cm} \leqslant \sigma_{z} \leqslant 0.003 \mathrm{~cm}$ in regions corresponding to zero displacement, with an apparently more favourable range for $S / \sigma_{z}$ of $50 \leqslant\left(S / \sigma_{z}\right) \leqslant 80$. However, the final displacement fields obtained using the pattern matching method are averages calculated from eleven measured displacement fields obtained from a sequence of different random patterns. Hence, one might expect the noise level in these measurements to be less than those obtained from the attenuation technique which uses a single (time 


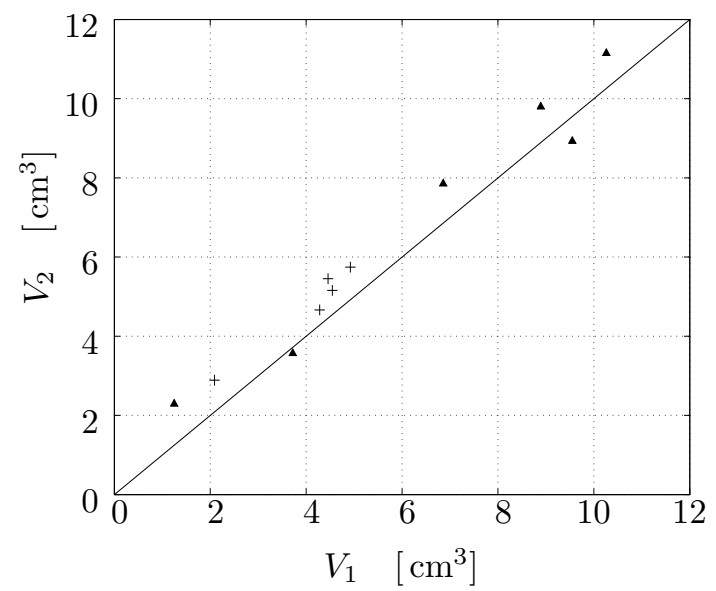

Figure 7: Plot comparing the volumes of deposited sediment, $V_{1}$, and eroded sediment, $V_{1}$, for the five experiments with $z_{r}=0.2 \mathrm{~cm}$ (crosses), and the six experiments with $z_{r}=0.8 \mathrm{~cm}$ (triangles).

averaged) image.

Moreover, as can be seen in figure 6, the mean profile obtained using the pattern matching method has much smoother features than that obtained from the attenuation method. This is because the pattern matching technique removes highest frequency components, with wavelengths less than the spacing of the interrogation window used in the pattern matching algorithm. The attenuation technique, however, uses each individual pixel which, for the high-resolution camera, resolves individual particle size scales.

Finally, a simple secondary level test may also be applied to the mean profiles $\langle\Delta z\rangle$ obtain using the attenuation technique. By mass conservation, the volume of eroded sediment must equal the volume of deposited sediment, and so

$$
\int_{0}^{r_{0}}\langle\Delta z\rangle r \mathrm{~d} r=0
$$

where $r_{0}$ is a suitably chosen value of $r$ beyond which $\langle\Delta z\rangle=0$. The deposited sediment volume, $V_{1}$, and the eroded sediment volume, $V_{2}$, were defined respectively by

$$
\begin{aligned}
& V_{1}=\int_{0}^{r_{0}}\langle\Delta z\rangle^{+} r \mathrm{~d} r, \\
& V_{2}=\int_{0}^{r_{0}}\langle\Delta z\rangle^{-} r \mathrm{~d} r,
\end{aligned}
$$

where $\langle\Delta z\rangle^{+}=\{\langle\Delta z\rangle:\langle\Delta z\rangle>0\}$, and $\langle\Delta z\rangle^{-}=\{\langle\Delta z\rangle:\langle\Delta z\rangle<0\}$. Figure 
7 shows a comparison of $V_{1}$ plotted against $V_{2}$ for each of the five experiments performed with $z_{r}=0.2 \mathrm{~cm}$ (crosses), and also each of the six experiments with $z_{r}=0.8 \mathrm{~cm}$ (triangles). For both sets of experiments, the smallest and largest eroded volumes correspond to the impact craters produced by vortex rings with $R e \approx 5000$ and $R e \approx 28000$ respectively. The same comparison was performed on the measurements obtained in Munro et al. (2004) (see figure 7 of that paper), with an equivalent degree of correspondence between $V_{1}$ and $V_{2}$.

\subsection{High-speed camera images}

So far, the attenuation method described has been restricted to measuring characteristics of the crater in its final state, i.e. when any resuspended sediment has been redeposited. However, to perform an analysis of the dynamics of the interaction between the approaching vortex ring and the particle layer, it is necessary to have detailed measurements for the evolution of the impact area as the interaction occurs. In this subsection we illustrate how the attenuation technique is capable of obtaining such measurements. Recall that this article is not intended as a detailed analysis of the interaction process, but presents the details of the attenuation technique used in the analysis. However, it will be useful to briefly describe some details of the interaction, and in particular a description of the features we intend to measure. Further details of the interaction can be found in Munro \& Dalziel (2003).

As the vortex ring approaches the particle layer, the potential-like flow induced in the surrounding fluid produces a radial shear stress at the layer surface, centred below the stagnation point at the leading face of the vortex ring. The magnitude of this stress increases as the ring gets closer to the layer. It was observed that, for particles used in our experiments and the range of Re considered, the stress is sufficient to start displacing the particles radially outwards when the ring is of order $R$ above the particle layer. As the ring gets closer more particles are displaced, which results in a congregation of displaced particles at the edge of the region of significant stress. This process is illustrated by the sequence of images shown in figure $8(\mathrm{a})-(\mathrm{c})$, which show the crater development with the initial bed depth at $z_{r}=0.8 \mathrm{~cm}$ and a vortex ring with $R e=2 \times 10^{4}$. Note that these initial stages of the interaction occur over very short timescales, for instance the sequence of images shown in figure 8 correspond to an interval of approximately $0.03 \mathrm{~s}$.

Eventually, provided $R e$ is large enough, a viscous-inviscid interaction takes place between the (separated) induced boundary layer and the outer flow, full details of which (in the case of a solid boundary) can be found in Walker et al. (1987). This results in an ejection of vortical fluid from the boundary layer, which here creates a high concentration splash of entrained particles in the process. The ejection of vortical fluid is an effective resuspension mechanism, 

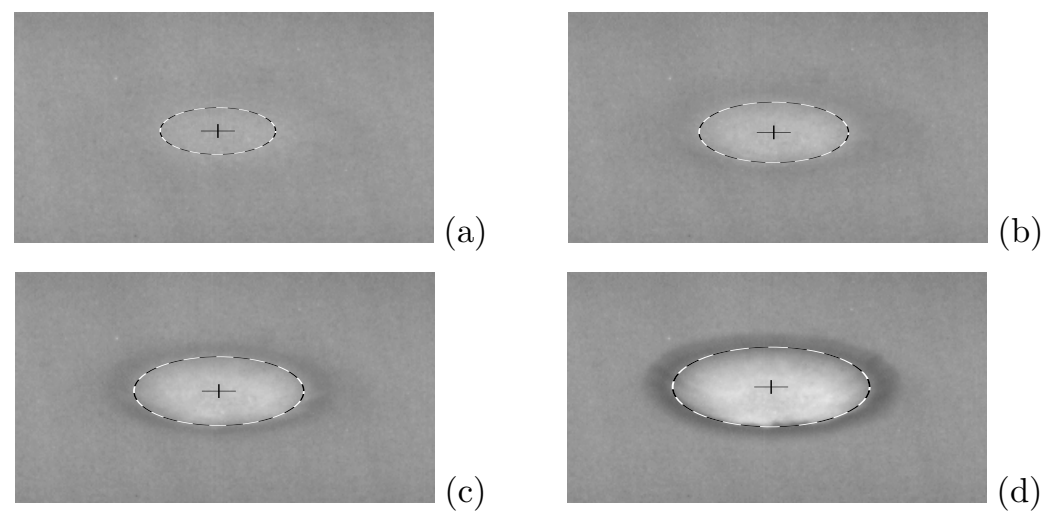

Figure 8: A sequence of scaled intensity fields, $I(x, y, t)$, showing the crater development with $z_{r}=0.8 \mathrm{~cm}$ and $R e=2 \times 10^{4}$. Image (a) shows the initial stages through to image (d) which shows the particle splash beginning to develop. The crosses and broken-line circles superimposed on top of the (oblique view) images show the location of the crater centre and the corresponding measured values of $R_{1}(t)$ respectively. The time-step between each image is approximately $0.01 \mathrm{~s}$.

and is able to carry much of the entrained material distances of order several $R$ above the layer. The image shown in figure $8(\mathrm{~d})$ corresponds to the initial stages of the splash development.

Note that entrained particles transported by the ejected vortical fluid are suspended in the fluid above the impact area, thus obstructing the camera view. As a result the attenuation method described here is unable to provide reliable measurements of the impact area in the interval between the occurrence of the splash and when any suspended material has fallen out of suspension. As a result, attention is henceforth restricted to the period prior to the occurrence of the splash.

One important measure required to describe this period of the crater evolution (represented by the sequence of images in figure 8(a)-(d)), as the surface stress develops, is the growth of the crater radius . For example, in figure 8(c), this radius is defined by the inner edge of darkened ring region, corresponding to the radial particle mound, where $\Delta z(r, \theta)=0$. Of course, additional, equally important features will also be required to characterize the crater development. However, in the remainder of this section we limit discussion to how the processed images were used to measure the initial growth of this crater radius. To be consistent with the notation adopted in Munro et al. (2004), the radius will be denoted by $R_{1}(t)$. It is worth noting that the radius of the crater can continue to increase after the splash, while the vortex structure remains coherent and continues to interact with particle layer. 
Again, the processing procedure was applied separately to the images obtained for the $z_{r}=0.2 \mathrm{~cm}$ and $z_{r}=0.8 \mathrm{~cm}$ cases, using the appropriate coordinate transformations and calibration curve. So that equation (6) could be applied, the sequence of scaled intensity fields,

$$
I(x, y, t)=\frac{P\left(x, y, t+t_{0}\right)}{\bar{P}\left(x, y ; z_{0}\right)} \quad\left(0 \leqslant t \leqslant t_{s}\right),
$$

were generated, where $P\left(x, y, t_{0}\right)$ is an image of the particle layer just prior to the initial crater formation, and $P\left(x, y, t_{s}+t_{0}\right)$ is the image in which the onset of the splash is first observed. As before, $\bar{P}\left(x, y ; z_{0}\right)$ denotes the averaged intensity field generated from the image of the illuminated tank floor. The scaled intensity fields were substituted into (6) and the results used to generate the displacement fields, $\Delta z(x, y, t)=z(x, y, t)-z_{r}$.

For each of the experiments performed, estimates for the crater diameter $2 R_{1}$ were easily obtained using the displacement fields, $\Delta z(x, y, t)$, at each time step $t_{i}$ in the interval $0 \leqslant t \leqslant t_{s}$. The estimates were obtained by scanning through $\Delta z(x, y, t)$ in the reverse direction and identifying the diameter as the distance between appropriate regions where $\Delta z$ goes from being negative to positive. The results of this procedure for $z_{r}=0.2 \mathrm{~cm}$ and $z_{r}=0.8 \mathrm{~cm}$ are shown in figure 9 (a) and (b) respectively. In figure 9 the crater radius $R_{1}$ has been nondimensionalized by the outlet radius $R$, and time $t$ by the vortex ring turn-over time $R / U$. Note that also shown in each of the images in figure 8 is the location of the crater centre (crosses), and the corresponding measured values of $R_{1}$ (broken line circles) relative to the crater centre.

For both $z_{r}=0.2 \mathrm{~cm}$ and $z_{r}=0.8 \mathrm{~cm}$, the separation and resuspension occur at approximately $R_{1}=R$, limiting the range of data plotted in figure 9 as the attenuation technique fails once there are particles in suspension. However, as noted previously, the crater radius continues to increase after the separation, provided the vortex ring remains coherent and continues to interact with the particle layer. Analysis of the displacement fields $\Delta z(x, y)$ obtained in section 4.1 showed that, for the final crater state, $R_{1}$ is of order $3 R$ and $2 R$ for $z_{r}=$ $0.2 \mathrm{~cm}$ and $z_{r}=0.8 \mathrm{~cm}$ respectively.

The initial layer depth $z_{r}$ seems to have little effect on the radius at which the separation occurs. It does, however, have a substantial effect on the time at which it occurs. Figure 9 shows that for the $z_{r}=0.2 \mathrm{~cm}$ case, and over the range of $R e$ considered, separation occurs at values of $\tau=t U / R$ in the range $0.6 \leqslant \tau \leqslant$ 1.2. For the $z_{r}=0.8 \mathrm{~cm}$ case the corresponding range for the (nondimensional) separation times is $0.5 \leqslant \tau \leqslant 2.3$. That is, at the lower range values of $R e$ there is little difference in the separation time between the $z_{r}=0.2 \mathrm{~cm}$ and $z_{r}=0.8 \mathrm{~cm}$ cases. However, at the high range values $R e$ the separation time for the $z_{r}=0.8 \mathrm{~cm}$ case is effectively twice that of corresponding separation time for $z_{r}=0.2 \mathrm{~cm}$. This implies that the degree of layer deformation during the 


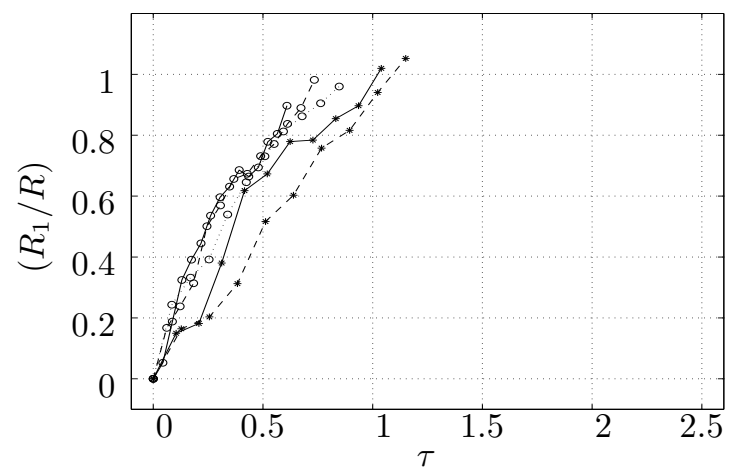

(a)

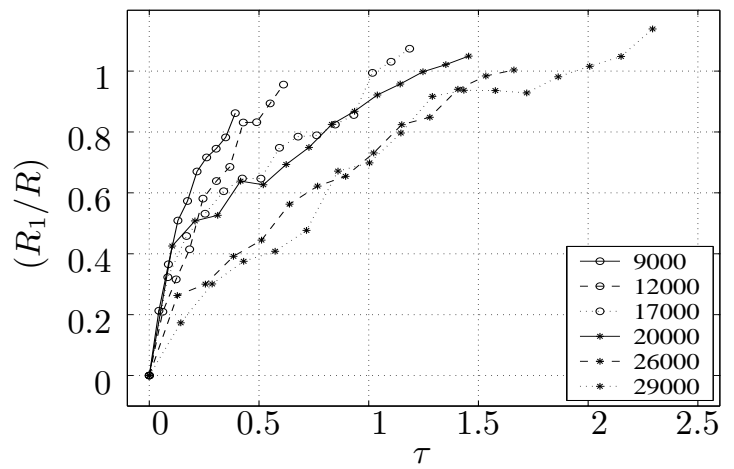

(b)

Figure 9: Plot showing the development of $R_{1}$, nondimensionalized by the outlet radius $R=$ $1.95 \mathrm{~cm}$, for (a) $z_{r}=0.2 \mathrm{~cm}$ and (b) $z_{r}=0.8 \mathrm{~cm}$, where $\tau=t U / R$ is the nondimensionalized time $(R / U$ is the vortex ring turn-over time). Note that the time step between consecutive points is $\Delta t=t_{i+1}-t_{i} \approx 0.0038 \mathrm{~s}$. The legend in (b), showing the relevant values of $R e$, applies to both plots.

crater formation significantly effects the vortex ring and separation dynamics.

\section{Discussion}

This paper has presented a non-intrusive method for obtaining accurate high-resolution measurements of sediment redeposition levels at camera-resolution scales over the whole domain of interest. Furthermore, the method is also capable of providing high-resolution measurements of the sediment layer thickness as a function of time (over time scales determined by the frame rate of the camera used), at least while the suspended particle load remains insignificant.

The simplicity of the system setup, and relatively inexpensive equipment required, means that the attenuation technique is an attractive alternative to 
current, more expensive systems for measuring sediment displacements levels in the laboratory. There are, however, several potential restrictions. Firstly, the particles and the base of the container used must be transparent (unlike the pattern matching method where opaque particles are the preference). Secondly, the particles need to be of small dimension with a narrow size distribution (so that the assumption of statistically homogeneous packing can be made). The measurements presented here indicate this assumption is certainly valid for deep particle layers where, in this specific instance, self-similarity was achieved for $z \gtrsim 18 d$. The self-similarity breaks down in the zero depth limit, and an alternative attenuation model is suggested for this case. Furthermore, recall that the operational dynamic range of the method may restrict the vertical resolution of the displacement measurements obtained (see section 3 for a detailed discussion). This was not an issue in the experiments described here where a dynamic range of $z \lesssim 40 d$ allowed vertical displacements of the order of $d$ to be achieved; however, this issue may become significant for experiments which use much greater initial bed depths.

One may, superficially, assume that the optimal setup of the apparatus would be the opposite of that presented, i.e. to view the particle layer from below the tank, and illuminate from above. Indeed, this setup was implemented in our initial efforts. It quickly, however, becomes evident that such an arrangement is not useful except for very thin layers. As the structure of the crater appears at the upper surface of the particle layer, a bottom-up view means that the crater structure is viewed through the underlying (undisturbed) particles. The presence of the underlying particles acts to diffuse the image of the crater structure, effectively filtering out the fine scale components of the crater structure.

Through direct comparison, the attenuation method presented was found to provide an equivalent degree of accuracy to that of the pattern matching technique described in Munro et al. (2004). Despite the continuum assumptions used for the calibration, the method is able to measure layer displacements comparable with the particle diameter $d=0.025 \mathrm{~cm}$. In addition, the attenuation technique does not suffer from the problems associated with individual pattern structures becoming hidden from view, or the averaging of the high wavenumber, small scale structures that occurs with the pattern matching technique.

\section{Acknowledgements}

The authors would like to thank David Page-Croft for technical assistance while assembling the experimental equipment. Munro was provided with financial support by the EPSRC (grant number GR/R25620/01) and the Isaac 
Newton Trust.

\section{References}

Best J; Ashworth P (1994) A high-resolution ultrasonic bed profiler for use in laboratory flumes. J Sedimentary Res 24: 279-288.

Dingler JR; Boylls JC; Lowe RL (1977) A high frequency sonar for profiling small scale subaqueous bedforms. Marine Geology 24: 279-288.

Glezer A (1988) The formation of vortex rings. Phys Fluids 31: 3532-3541.

Hancock G; Willgoose G (2001) The production of digital elevation models for experimental model landscapes. Earth Surf Processes Landforms 26: 475-490.

Irish JL; White TE (1998) Coastal engineering applications of high resolution Lidar bathymetry. Coastal Eng 35: 47-71.

Lague D (2004) Private Communication.

Lague D; Crave A; Davy P (2003) Laboratory experiments simulating the geomorphic response to tectonic uplift. J Geophys Res 108(B1): 2008.

Leppinen DM; Dalziel SB (2001) A light attenuation technique for void fraction measurement of microbubbles. Exp in Fluids 30: 214-220.

Linden PF; Turner JS (2001) The formation of 'optimal' vortex rings, and the efficiency of propulsion devices. J Fluid Mech 427: 61-72.

Measures RM (1984) Laser Remote Sensing: Fundamentals and Applications. Wiley and Sons.

Munro RJ; Dalziel SB (2003) Particle resuspension by an impacting vortex ring. In Sediment and Sediment Transport, Kluwer Academic Publishers: 105-108.

Munro RJ; Dalziel SB, Jehan H (2004) An pattern matching technique for measuring sediment redeposition levels. Exp in Fluids 37: 399-408.

Rooij F de; Dalziel SB; Linden PF (1999) Electrical measurement of sediment layer thickness under suspension flows. Exp in Fluids 26: 470-474.

Richards KS; Robert A (1986) Laboratory experiments with the HR multipurpose profile follower on a rippled sad bed. University of Cambridge, Department of Geography Working Paper, 22 p.

Richardson EV; Simons DB; Posakony GJ (1961) Sonic depth sounder for laboratory and field use. United States Geological Survey Circular 450, 7 p.

Walker JDA; Smith CR; Cerra AW; Doligalski TL (1987) The impact of a vortex ring on a wall. J Fluid Mech 181: 99-140.

Williams KL; Jackson DR (1998) Bistatic bottom scattering: model, experiments, and model/data comparison. J Acoustical Soc Am 103: 169-181. 